\title{
Machine learning-based prediction model for responses of bDMARDs in patients with rheumatoid arthritis and ankylosing spondylitis
}

Seulkee Lee ${ }^{1}$, Seonyoung Kang ${ }^{1}$, Yeonghee Eun ${ }^{1}$, Hong-Hee Won ${ }^{2}$, Hyungjin Kim', Jaejoon Lee ${ }^{1}$, Eun-Mi Koh ${ }^{1}$ and Hoon-Suk Cha ${ }^{1 *}$ (D)

\begin{abstract}
Background: Few studies on rheumatoid arthritis (RA) have generated machine learning models to predict biologic disease-modifying antirheumatic drugs (bDMARDs) responses; however, these studies included insufficient analysis on important features. Moreover, machine learning is yet to be used to predict bDMARD responses in ankylosing spondylitis (AS). Thus, in this study, machine learning was used to predict such responses in RA and AS patients.

Methods: Data were retrieved from the Korean College of Rheumatology Biologics therapy (KOBIO) registry. The number of RA and AS patients in the training dataset were 625 and 611, respectively. We prepared independent test datasets that did not participate in any process of generating machine learning models. Baseline clinical characteristics were used as input features. Responders were defined as those who met the ACR 20\% improvement response criteria (ACR20) and ASAS 20\% improvement response criteria (ASAS20) in RA and AS, respectively, at the first follow-up. Multiple machine learning methods, including random forest (RF-method), were used to generate models to predict bDMARD responses, and we compared them with the logistic regression model.

Results: The RF-method model had superior prediction performance to logistic regression model (accuracy: 0.726 [95\% confidence interval (CI): 0.725-0.730] vs. 0.689 [0.606-0.717], area under curve (AUC) of the receiver operating characteristic curve (ROC) 0.638 [0.576-0.658] vs. 0.565 [0.493-0.605], F1 score 0.841 [0.837-0.843] vs. 0.803 [0.7320.828], AUC of the precision-recall curve 0.808 [0.763-0.829] vs. 0.754 [0.714-0.789]) with independent test datasets in patients with RA. However, machine learning and logistic regression exhibited similar prediction performance in AS patients. Furthermore, the patient self-reporting scales, which are patient global assessment of disease activity (PtGA) in RA and Bath Ankylosing Spondylitis Functional Index (BASFI) in AS, were revealed as the most important features in both diseases.
\end{abstract}

Conclusions: RF-method exhibited superior prediction performance for responses of bDMARDs to a conventional statistical method, i.e., logistic regression, in RA patients. In contrast, despite the comparable size of the dataset,

\footnotetext{
*Correspondence: hoonsuk.cha@samsung.com

${ }^{1}$ Department of Medicine, Samsung Medical Center, Sungkyunkwan

University School of Medicine, 81 Irwon-ro, Gangnam-gu, Seoul 06351, Republic of Korea

Full list of author information is available at the end of the article
} permits use, sharing, adaptation, distribution and reproduction in any medium or format, as long as you give appropriate credit to the original author(s) and the source, provide a link to the Creative Commons licence, and indicate if changes were made. The images or other third party material in this article are included in the article's Creative Commons licence, unless indicated otherwise in a credit line to the material. If material is not included in the article's Creative Commons licence and your intended use is not permitted by statutory regulation or exceeds the permitted use, you will need to obtain permission directly from the copyright holder. To view a copy of this licence, visit http://creativecommons.org/licenses/by/4.0/. The Creative Commons Public Domain Dedication waiver (http://creativeco mmons.org/publicdomain/zero/1.0/) applies to the data made available in this article, unless otherwise stated in a credit line to the data. 
machine learning did not outperform in AS patients. The most important features of both diseases, according to feature importance analysis were patient self-reporting scales.

Keywords: Rheumatoid arthritis, Ankylosing spondylitis, Machine learning, TNFi

\section{Background}

Biologic disease-modifying antirheumatic drugs (bDMARDs) play a pivotal role in the treatment of various rheumatologic diseases, such as rheumatoid arthritis (RA) and ankylosing spondylitis (AS), particularly those resistant to conventional synthetic disease-modifying rheumatic drugs (csDMARDs). However, approximately $30 \%$ and $20 \%$ of RA [1,2] and AS [3-5] patients, respectively, do not respond well to their initial bDMARD therapy. A few months are required to determine the efficacy of the medications. Non-responders could experience high drug costs, unimproved disease conditions, and side effects during this period [6-9]. Therefore, methods to predict the responses prior to the start of bDMARDs is garnering substantial interest.

Several studies simply identified and compared clinical factors, such as sex, age, disease duration, and disease activity, in both diseases to influence the treatment responses of bDMARDs [10,11], rather than making a predictive model. Because the relationship between clinical variables and phenotypes is complex, machine learning methods outperform conventional statistical models in predicting clinical outcomes in various circumstances [12-15]. Recently, the use of machine learning to predict anti-tumor necrosis factor (TNFi) drug responses in RA patients has been published [16], based on the largest data obtained among machine learning studies conducted to date in RA. However, the study did not include much about feature importance analysis. In the case of AS, although machine learning to predict early TNFi users was conducted previously [17], no machine learning model has been developed to predict the responses of bDMARDs.

This study aims to examine whether machine learning can better predict the treatment responses of bDMARDs than conventional statistical methods. In addition, this study aims to identify important clinical factors that affect the treatment responses of bDMARDs through machine learning. Machine learning models including random forest (RF-method), extreme gradient boosting (XGBoost), artificial neural network (ANN), and support vector machine (SVM), are presented to predict bDMARD responses in patients with RA and AS, respectively. The prediction performances between machine learning methods, as well as with a conventional statistical method, which is logistic regression, were compared. Next, feature importance analysis was performed with the generated machine learning models to delineate the factors that are important in training models.

\section{Methods}

\section{Data acquisition and participants}

The data for this study were retrieved from the Korean College of Rheumatology Biologics therapy (KOBIO) registry [18], a prospective nationwide biologic therapy registry for RA, AS, and psoriatic arthritis, which includes 45 hospitals in South Korea. This registry enrolled patients who started bDMARDs with baseline clinical data and followed up annually. Our target cohort population included patients (1) with RA or AS who enrolled in the registry between December 2012 and February 2019, (2) who started bDMARDs for the first time, and (3) who were followed up for 1 year or more. All patients met the 1987 American College of Rheumatology (ACR) criteria or the 2010 ACR/European League Against Rheumatism (EULAR) criteria for RA patients, or the modified New York criteria for AS or the assessment of spondyloarthritis international society (ASAS) axial spondyloarthritis criteria for AS patients. Only bDMARD-naïve patients were included to maintain the homogeneity of the population. Patients who did not have baseline clinical data or could not check the 1-year treatment response were excluded.

The data were divided into training and independent test datasets by region of hospitals (Additional file 1: Text S1). Predictive models were generated using machine learning with only the training dataset. The independent test dataset did not participate in any training or internal validation of predictive models. It was only used for the final external validation of each trained model. Because every hospital had independent researchers and laboratory facilities and maintained individual clinical practices, we expected that dividing the test dataset by enrolled hospitals would serve similar to the independent cohort dataset. Table 1 lists the number of individuals included in each dataset.

\section{Model design Input features}

The KOBIO registry collects data on demographics, comorbidities, disease activity, medication (bDMARDs, and concomitant or previous use of csDMARDs), image, extra-articular features, functional assessment, and laboratory findings as baseline clinical characteristics. We 
Table 1 Number of training and test datasets in patients with RA and AS

\begin{tabular}{lll}
\hline & Training dataset & $\begin{array}{l}\text { Independent } \\
\text { test dataset }\end{array}$ \\
\hline RA & 625 & 322 \\
AS & 611 & 296 \\
\hline
\end{tabular}

filtered input features that included only sparse information as using too many input features results in overfitting. The numbers of selected input features were 74 and 75 in RA and AS, respectively (Additional file 3: Table S1).

\section{Training prediction models}

Using a clinical data matrix, we trained the prediction models to distinguish between bDMARD responders and non-responders. Patients who met the ACR 20\% improvement response criteria (ACR20) [19] or ASAS $20 \%$ improvement response criteria (ASAS20) [20] for RA or AS, respectively, were classified as responders. The remaining patients were classified as non-responders. ACR20 and ASAS20 have been frequently used as treatment response measures in clinical trials [21-23]. Because each input feature has a different scale, continuous features are normalized to a range of $0-1$ to match the value of the categorical features, which were used directly. RF-method, XGBoost, ANN, and SVM were used to train prediction models to classify patients as responders or non-responders. In addition, a logistic regression model was constructed as a representative of a conventional statistical method to compare machine learning models. RF-method [24], XGBoost [25], and ANN [26] have several hyperparameters that must be determined before training. However, there is no consensus on the hyperparameters that are suitable for predicting clinical prognosis. Therefore, multiple machine-learning models were tested by varying the hyperparameters. The hyperparameters for RF-method include the maximum depth of a tree, total number of trees, minimum sample split, and minimum leaf samples. In the case of XGBoost, the hyperparameters include the maximum depth of a tree, learning rate, and gamma value. For the ANN, the hyperparameters include the number of hidden layers and nodes and the learning rate. The learning rate is the number of changes that newly acquired information undergoes while overriding old information, gamma refers to the minimum loss reduction required to make a further partition on a leaf node of the tree, the minimum sample split refers to the minimum number of samples required to split an internal node, and the minimum leaf samples refer to the minimum number of samples required to be at a leaf node.
We chose hyperparameters with the best performance and those that performed better than logistic regression in all respects. Our training codes and generated prediction models have been made publicly accessible (https:// github.com/SeulkeeLee123/KOBIO_biologics).

\section{Performance evaluation}

The prediction models were evaluated in three rounds of threefold cross-validation [27]. Because the responders and non-responders were unevenly distributed in the dataset, stratified cross-validation was used to divide the dataset. As mentioned earlier, only "training dataset" was used to generate prediction models. In each round, the training dataset was randomly divided into three equal sizes with stratified probability. A model was trained on two of these parts and scored on one remainder. This process was repeated thrice. Three rounds of tests resulted in a total of nine scores, and the average was used as the estimated performance score of the model. Finally, the generated models were tested with a pre-divided "independent test dataset" for external validation. The performance was measured by the accuracy, area under curve (AUC) of a receiver operating characteristic curve (ROC) and precision-recall curve, and F1 score. In addition, we used bootstrapping to calculate the confidence interval of performances [28]. A total of 1000 bootstrap iterations were used by sampling with replacement. For a confidence interval of $95 \%$, the values at the 2.5 percentile and 97.5 percentile were selected as the lower and upper bounds, respectively.

\section{Feature importance analysis}

Machine learning methods provide feature importance analysis, which can reveal important clinical features to predict treatment responses. For RF-method and XGBoost, the Gini importance was used for the feature importance analysis. However, compared to other machine learning methods, identifying the importance of each feature in ANN is more difficult because of its "black box" characteristics. There are several methods for evaluating feature importance despite the limitations $[29,30]$. We used the differential value of the prediction score in changing each input for feature importance. In previous studies, this method was called "risk backpropagation" [30]. Furthermore, we performed analysis using clinical factors reported as important based on the feature importance analysis to evaluate whether additional clinical significance can be inferred from the results. The detailed methods for feature importance analysis are presented in the Text S1. 


\section{Prediction models of each bDMARDs}

Separate prediction models were developed for patients who use specific bDMARDs to determine the differences in models and feature importance by varying the medications. The number of medication users less than 50 were excluded from the individual analysis owing to their small size. Consequently, abatacept, adalimumab, etanercept, infliximab, and tocilizumab were chosen for RA; adalimumab, etanercept, golimumab, and infliximab were chosen for AS. An identical methodology was used to generate and evaluate the prediction models when using the entire data.

\section{Statistical analysis}

Python (ver. 3.8.6) and R (ver. 3.6.3) [31] were used for statistical analysis. All machine learning models were generated and evaluated using the Python code.
Scikit-learn (ver. 0.24.1) [32] module was used for the RFmethod, SVM, and logistic regression models; xgboost (ver. 1.3.3) [25] was used for the XGBoost models. Tensorflow (2.4.1) [33] was used for the ANN models.

\section{Results}

\section{Demographic and characteristics of the patients}

The number of RA and AS patients included in the training dataset were 625 and 611, respectively. The demographic and baseline clinical characteristics are summarized in Tables 2 and 3, respectively. The RA and AS patients were divided into responders and nonresponders, indicating those who achieved ACR20 and ASAS20 and those who did not, respectively. In the case of RA patients, responders exhibited a higher disease activity (swollen joint count [SJC] 7.00 vs. 5.87, $p=0.017$; tender joint count $[\mathrm{TJC}] 8.78$ vs. $7.68, p=0.045$; patient

Table 2 Demographics and baseline clinical characteristics of the RA patients

\begin{tabular}{|c|c|c|c|c|}
\hline Baseline characteristics & Overall $n=625$ & Responder $n=466$ & Non-responder $n=159$ & $p$-value \\
\hline Sex (male, \%) & $103(16.5)$ & $80(17.2)$ & $23(14.5)$ & 0.503 \\
\hline Age at baseline (years) & $54.04(12.44)$ & $54.13(12.40)$ & $53.78(12.61)$ & 0.757 \\
\hline Disease duration (years) & $6.78(7.19)$ & $6.66(7.20)$ & $7.14(7.17)$ & 0.464 \\
\hline Height $(\mathrm{cm})$ & $159.41(7.01)$ & $159.50(7.08)$ & $159.16(6.81)$ & 0.606 \\
\hline Weight (kg) & $57.30(9.72)$ & $57.08(9.45)$ & $57.93(10.47)$ & 0.341 \\
\hline SJC & $6.71(5.18)$ & $7.00(5.10)$ & $5.87(5.36)$ & 0.017 \\
\hline $\mathrm{TJC}$ & $8.50(5.98)$ & $8.78(5.98)$ & $7.68(5.93)$ & 0.045 \\
\hline PtGA & $7.24(1.78)$ & $7.47(1.69)$ & $6.55(1.87)$ & $<0.001$ \\
\hline PhGA & $6.56(1.75)$ & $6.69(1.72)$ & $6.18(1.78)$ & 0.001 \\
\hline RAPID3 & $15.54(5.68)$ & $16.10(5.70)$ & $13.89(5.30)$ & $<0.001$ \\
\hline $\mathrm{ESR}(\mathrm{mm} / \mathrm{h})$ & $46.63(25.66)$ & $46.38(25.98)$ & $47.36(24.77)$ & 0.678 \\
\hline $\operatorname{CRP}(\mathrm{mg} / \mathrm{dL})$ & $2.34(3.16)$ & $2.40(3.00)$ & $2.16(3.59)$ & 0.405 \\
\hline HTN (\%) & $167(26.7)$ & $126(27.0)$ & $41(25.8)$ & 0.838 \\
\hline $\mathrm{DM}(\%)$ & $61(9.8)$ & $36(7.7)$ & $25(15.7)$ & 0.005 \\
\hline CKD (\%) & $5(0.8)$ & $3(0.6)$ & $2(1.3)$ & 0.814 \\
\hline Rheumatoid factor positive (\%) & $551(88.2)$ & $410(88.0)$ & $141(88.7)$ & 0.926 \\
\hline Anti-CCP positive (\%) & $494(79.0)$ & $373(80.0)$ & $121(76.1)$ & 0.346 \\
\hline Methotrexate (\%) & $531(85.0)$ & $400(85.8)$ & $131(82.4)$ & 0.357 \\
\hline Hydroxychloroquine (\%) & $175(28.0)$ & $136(29.2)$ & $39(24.5)$ & 0.304 \\
\hline Sulfasalazine (\%) & $85(13.6)$ & $67(14.4)$ & $18(11.3)$ & 0.403 \\
\hline Leflunomide (\%) & $179(28.6)$ & $127(27.3)$ & $52(32.7)$ & 0.226 \\
\hline Abatacept (\%) & $68(10.9)$ & $49(10.5)$ & 19 (11.9) & 0.723 \\
\hline Adalimumab (\%) & $168(26.9)$ & $121(26.0)$ & 47 (29.6) & 0.436 \\
\hline Etanercept (\%) & $94(15.0)$ & $64(13.7)$ & $30(18.9)$ & 0.151 \\
\hline Golimumab (\%) & $26(4.2)$ & $18(3.9)$ & $8(5.0)$ & 0.684 \\
\hline Infliximab (\%) & $64(10.2)$ & $40(8.6)$ & $24(15.1)$ & 0.029 \\
\hline Tocilizumab (\%) & $194(31.0)$ & $165(35.4)$ & $29(18.2)$ & $<0.001$ \\
\hline Tofacitinib (\%) & $11(1.8)$ & $9(1.9)$ & $2(1.3)$ & 0.835 \\
\hline
\end{tabular}

Data are shown in mean (standard deviation) if not otherwise specified

SJC swollen joint count, TJC tender joint count, PtGA patient global assessment of disease activity, PhGA physician global assessment of disease activity, RAPID3 routine assessment of patient index data 3, ESR erythrocyte sedimentation rate, CRP C-reactive protein, HTN hypertension, DM diabetes mellitus, CKD chronic kidney disease, anti-CCP anti-citrullinated protein 
Table 3 Demographics and baseline clinical characteristics of the AS patients

\begin{tabular}{|c|c|c|c|c|}
\hline Baseline characteristics & Overall $n=611$ & Responder $n=396$ & Non-responder $n=215$ & $p$-value \\
\hline Sex (male, \%) & $456(74.6)$ & $301(76.0)$ & $155(72.1)$ & 0.334 \\
\hline Age at baseline (years) & $38.76(13.29)$ & $37.88(12.85)$ & $40.38(13.95)$ & 0.026 \\
\hline Disease duration (years) & $3.56(5.23)$ & $3.43(4.99)$ & $3.80(5.63)$ & 0.405 \\
\hline Height (cm) & $169.24(8.46)$ & $169.95(8.37)$ & $167.93(8.47)$ & 0.005 \\
\hline Weight (kg) & $67.36(12.65)$ & $67.93(12.62)$ & $66.30(12.67)$ & 0.129 \\
\hline Peripheral arthritis (\%) & $234(38.3)$ & $155(39.1)$ & $79(36.7)$ & 0.621 \\
\hline Enthesitis (\%) & $120(19.6)$ & $84(21.2)$ & $36(16.7)$ & 0.222 \\
\hline Uveitis (\%) & $118(19.3)$ & $72(18.2)$ & $46(21.4)$ & 0.393 \\
\hline Dactylitis (\%) & $15(2.5)$ & $10(2.5)$ & $5(2.3)$ & 1.000 \\
\hline Psoriasis (\%) & $16(2.6)$ & $8(2.0)$ & $8(3.7)$ & 0.321 \\
\hline Inflammatory bowel disease (\%) & $8(1.3)$ & $6(1.5)$ & $2(0.9)$ & 0.814 \\
\hline \multicolumn{5}{|l|}{ Smoking } \\
\hline Smoker + ex-smoker & $179(45.2)$ & $284(46.5)$ & $105(48.8)$ & \multirow[t]{2}{*}{0.438} \\
\hline Non-smoker & $217(54.8)$ & $327(53.5)$ & $110(51.2)$ & \\
\hline BASDAI & $6.52(1.70)$ & $6.75(1.61)$ & $6.08(1.77)$ & $<0.001$ \\
\hline BASFI & $4.11(2.53)$ & $4.47(2.47)$ & $3.45(2.51)$ & $<0.001$ \\
\hline $\operatorname{ESR}(\mathrm{mm} / \mathrm{h})$ & $39.77(30.89)$ & $42.46(31.26)$ & $34.82(29.64)$ & 0.003 \\
\hline CRP (mg/dL) & $2.52(3.12)$ & $2.77(3.32)$ & $2.06(2.64)$ & 0.007 \\
\hline HTN (\%) & $96(15.7)$ & $48(12.1)$ & $48(22.3)$ & 0.001 \\
\hline DM (\%) & $18(2.9)$ & $11(2.8)$ & $7(3.3)$ & 0.934 \\
\hline CKD (\%) & $0(0.0)$ & $0(0.0)$ & $0(0.0)$ & NA \\
\hline HLA-B27 positive (\%) & $543(88.9)$ & $358(90.4)$ & $185(86.0)$ & 0.133 \\
\hline NSAIDs use (\%) & $521(85.3)$ & $334(84.3)$ & $187(87.0)$ & 0.449 \\
\hline Methotrexate (\%) & $44(7.2)$ & $23(5.8)$ & $21(9.8)$ & 0.100 \\
\hline Sulfasalazine (\%) & $35(5.7)$ & $19(4.8)$ & $16(7.4)$ & 0.246 \\
\hline Adalimumab (\%) & $253(41.4)$ & $165(41.7)$ & 88 (40.9) & 0.928 \\
\hline Etanercept (\%) & $74(12.1)$ & $47(11.9)$ & $27(12.6)$ & 0.905 \\
\hline Golimumab (\%) & $115(18.8)$ & $81(20.5)$ & $34(15.8)$ & 0.196 \\
\hline Infliximab (\%) & $169(27.7)$ & $103(26.0)$ & $66(30.7)$ & 0.253 \\
\hline
\end{tabular}

Data are shown in mean (standard deviation) if not otherwise specified.

BASDAI Bath Ankylosing Spondylitis Disease Activity Index, BASFI Bath Ankylosing Spondylitis Functional Index, ESR erythrocyte sedimentation rate, CRP C-reactive protein, HTN hypertension, DM diabetes mellitus, CKD chronic kidney disease, HLA human leukocyte antigen, NSAIDs non-steroidal anti-inflammatory drugs

global assessment of disease activity [PtGA] 7.47 vs. 6.55 , $p<0.001$; physician global assessment of disease activity [PhGA] 6.69 vs. $6.18, p=0.001$; routine assessment of patient index data 3 [RAPID3] score 16.10 vs. $13.89, p$ $<0.001)$, lower proportion of infliximab users (40 [8.6\%] vs. 24 [15.1\%], $p=0.029$ ), and higher proportion of tocilizumab users (165 [35.4\%] vs. 29 [18.2], $p<0.001)$ than non-responders. Meanwhile, in the case of AS patients, responders exhibited a higher disease activity (Bath Ankylosing Spondylitis Disease Activity Index [BASDAI] 6.75 vs. $6.08, p<0.001$; BASFI 4.47 vs. $3.45, p<0.001$ ), including erythrocyte sedimentation rate (ESR, 42.46 $\mathrm{mm} / \mathrm{h}$ vs. $34.82 \mathrm{~mm} / \mathrm{h}, p=0.003)$ and C-reactive protein (CRP) level (2.77 mg/dL vs. $2.06 \mathrm{mg} / \mathrm{dL}, p=0.007)$ than non-responders. In addition, responders were younger
(37.88 years vs. 40.38 years, $p=0.026)$ and taller $(169.95$ cm vs. $167.93 \mathrm{~cm}, p=0.005$ ).

\section{Prediction model optimization}

Prediction models that classified patients as responders or non-responders were trained using RF-method, XGBoost, ANN, SVM, and logistic regression (Fig. 1). The RF-method, XGBoost, and ANN models were significantly different in terms of their hyperparameters. Thus, we trained them repeatedly to determine an appropriate hyperparameter set for the input dataset (Additional file 2: Figures S1-6). Hyperparameters of better performing models than the logistic regression model were selected in terms of all four performance measures (accuracy, AUC of ROC curve, F1 score, and AUC of 
KOBIO registry

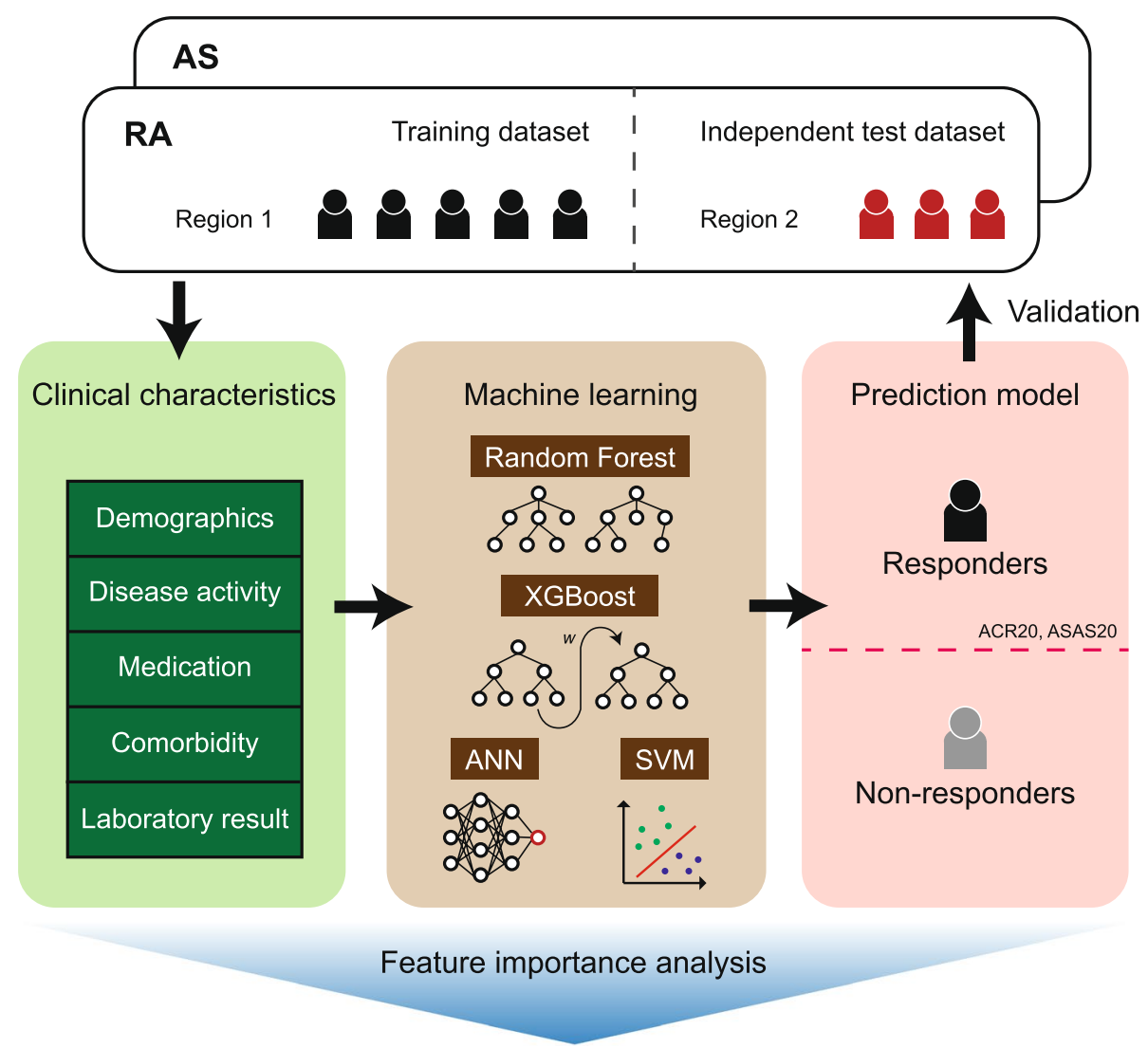

Fig. 1 Overview of the prediction model for responses of bDMARDs. The model uses data on baseline clinical characteristics, including comorbidities, disease activity, medication, image, extra-articular features, functional assessment, and laboratory findings, to classify the patient as a responder or non-responder

precision-recall curve). The chosen hyperparameter sets of each model are listed in Additional file 3: Table S2.

\section{Performance of predicting bDMARDs responses}

Performance of various models was compared in terms of the accuracy, AUC of the ROC and precision-recall curves, and F1 score. The prediction models were evaluated in three rounds of three-fold cross-validation. In both disease cohorts, RF-method showed the best performance among the various methods in almost all fields (Fig. 2). However, the differences were within the confidence intervals calculated using bootstrap methods. Prediction models with RA patients exhibited better performance in general. Although the different structures of the training dataset could affect the performance of the prediction methods, it is likely that the performance differs in reality because all four measures were better in RA patients.

\section{Evaluation on independent test dataset}

To validate the performance of the prediction model, we excluded data on specific hospitals from the processes to formulate prediction models and the excluded data were used as an independent test dataset. The performance of previously obtained prediction models was evaluated with data from an independent test dataset. In the case of RA patients, the prediction performances of the RFmethod and XGBoost models were higher than those of the logistic regression model (Fig. 3a). RF-method and XGBoost showed similar performances in all four performance measures; however, RF-method exhibited more robust results with the bootstrap method. The RF-method model showed better prediction performance than the logistic regression model, even considering the $95 \%$ confidence interval calculated using bootstrap methods (accuracy 0.726 [95\% confidence interval (CI) 0.725-0.730] vs. 0.689 [0.606-0.717], AUC of the ROC 0.638 [0.576-0.658] vs. 0.565 [0.493-0.605], 


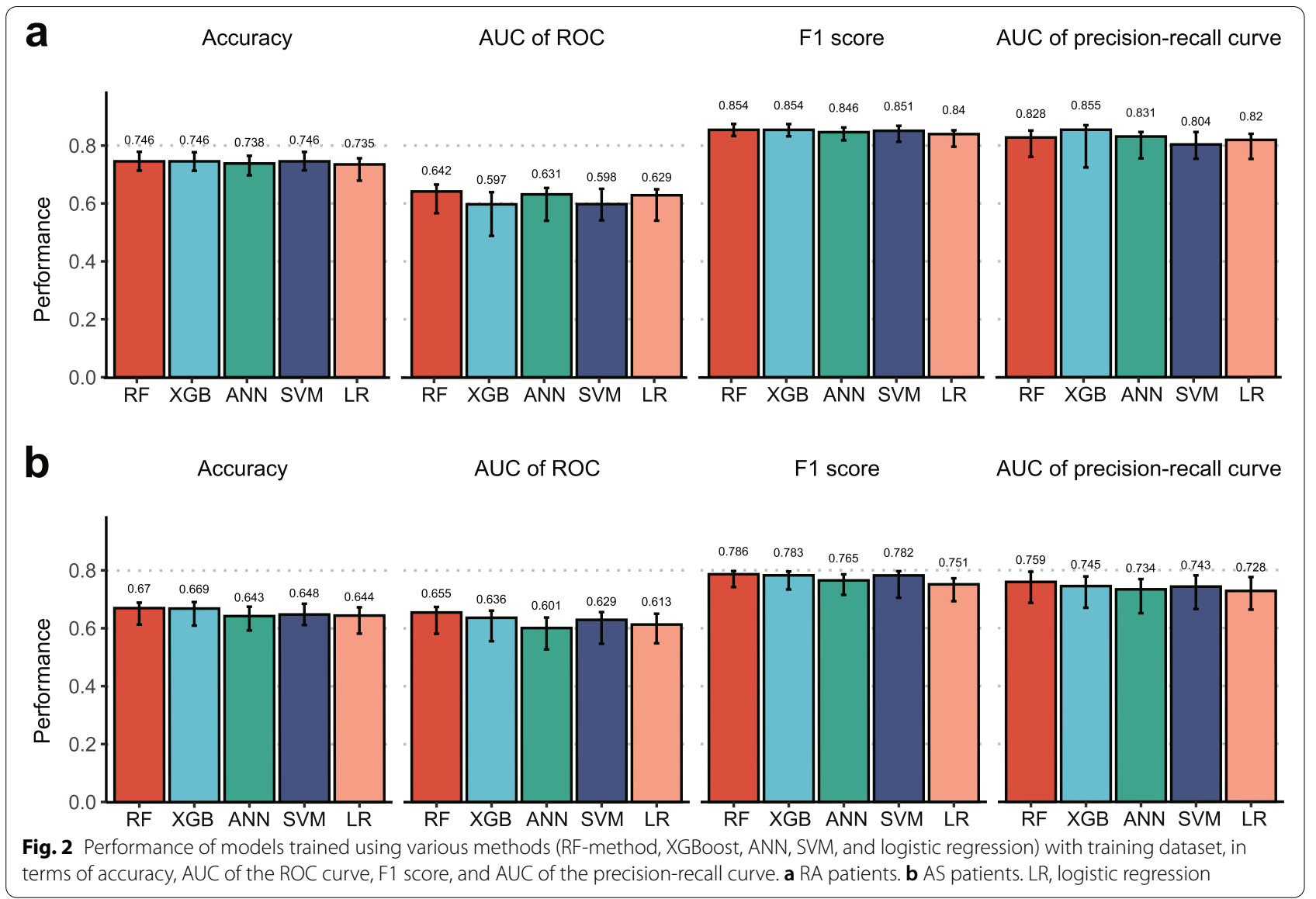

F1 score 0.841 [0.837-0.843] vs. 0.803 [0.732-0.828], AUC of the precision-recall curve 0.808 [0.763-0.829] vs. 0.754 [0.714-0.789]). The ANN and SVM did not show superior prediction performance. In contrast with RA patients, prediction performances between the machine learning methods and logistic regression in AS patients did not significantly differ (Fig. 3b).

\section{Feature importance analysis}

Feature importance analysis was implemented using the best-performing models of each RF-method, XGBoost, and ANN methods. Gini importance method was used for RF-method and XGBoost models, and risk backpropagation method was used for ANN models to calculate feature importance. The top three important input features of the RF-method model in RA patients were PtGA, RAPID3, and SJC (Fig. 4). Except for the ANN model in RA patients, the most important input feature was PtGA, which is a self-reported scale, rather than more objective features, such as laboratory results or physical examination. Among the three machine learning methods, ANN exhibited the worst prediction performance. In the feature importance analysis, the ANN showed significantly different results from the other two methods. Considering the prediction performance of the ANN model, the feature importance results of the ANN model were regarded as unreliable compared to other methods. In the case of AS, the most important input feature of all three machine learning methods was BASFI, which is a self-reported functional assessment score for AS (Fig. 4), followed by BASDAI in the RF-method and XGBoost models. By combining self-reported scales predicted to be important, we attempted to analyze whether additional information could be found through a conventional statistical method; however, the results were inconsistent (Text S1).

\section{Prediction models of different bDMARDs}

The training dataset was divided based on the type of bDMARD. RA patients were divided into abatacept, adalimumab, etanercept, infliximab, and tocilizumab users; AS patients were divided into adalimumab, etanercept, golimumab, and infliximab users. The performance of the RF-method and logistic regression models in RA patients did not differ from each other in all medication users (Additional file 2: Figure S7). The feature importance 


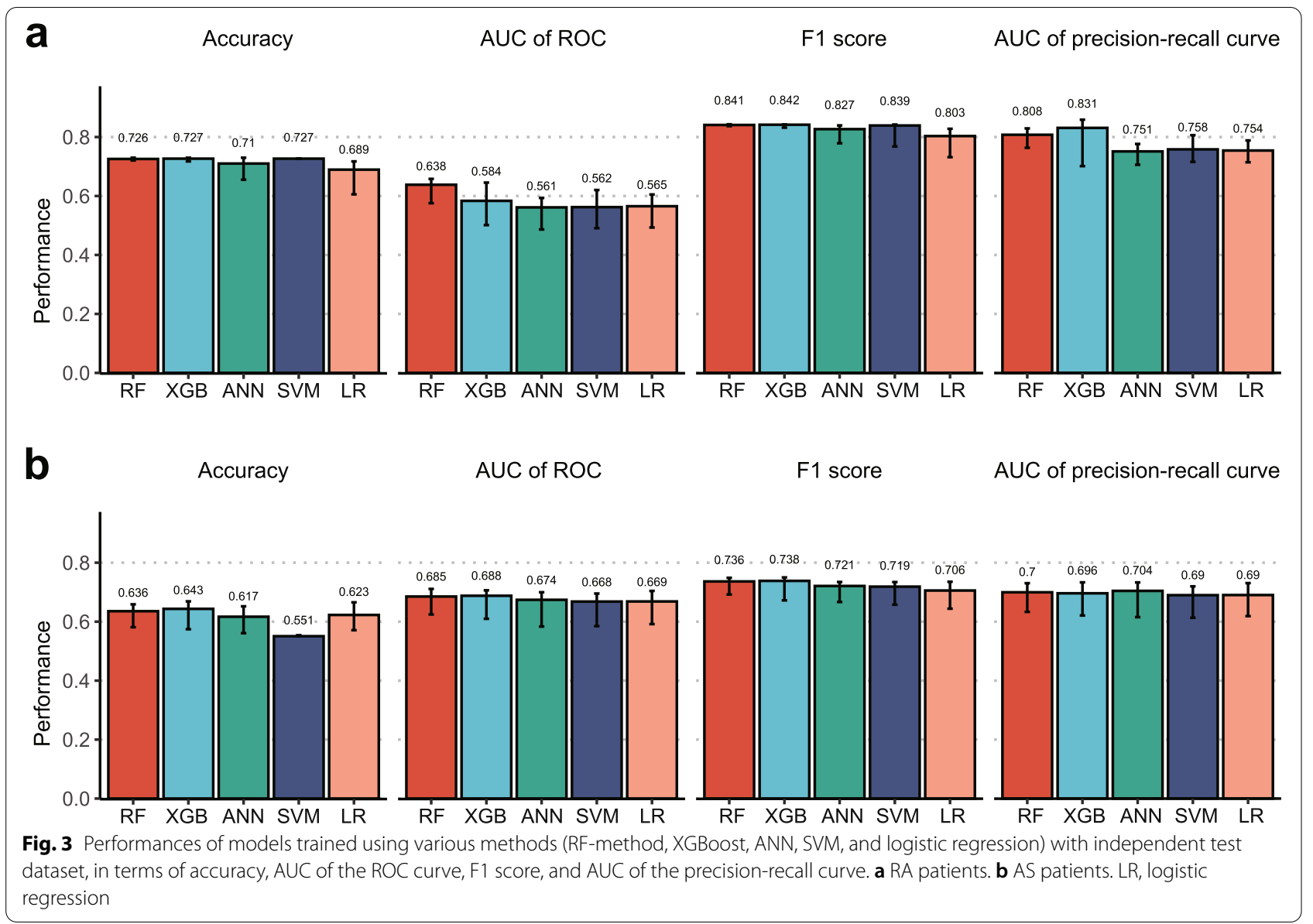

analysis results of the prediction models did not show consistent results for each medication cohort in the RA patients (Additional file 2: Figure S8). This could be because of the small size of the dataset of individual medication users. The performance of the RF-method models in AS patients showed similar results (Additional file 2: Figure S9). However, the RF-method prediction model in adalimumab users in AS patients showed better performance than the logistic regression model, particularly when the model was tested using an independent dataset, despite the borderline differences. Feature importance analysis of the RF-method model of adalimumab users in AS patients showed that the most important input feature was BASFI, followed by BASDAI (Additional file 2: Figure S10). This result was similar to that of the entire
AS dataset. The number of patients in the adalimumab cohort with AS was 253, the largest among the individual medication datasets. The size of the training dataset could be the reason for the better performance of the prediction model in the adalimumab cohort. In general, we could not formulate a prediction model for individual medication use with reasonable performance in most cases, and the primary reason seemed to be the size of the cohort.

\section{Discussion}

Various machine learning models were presented to classify the treatment responses of bDMARDs in RA and AS patients. In RA patients, RF-method was the most suitable method to predict treatment responses more

(See figure on next page.)

Fig. 4 Result of feature importance analysis from the best performing models of each machine learning method. The $X$-axis represents the input clinical features. The Y-axis represents the feature importance score calculated using the Gini importance or risk backpropagation methods in RF-method/XGBoost and ANN, respectively. The color of columns represents the categories in which the feature was included. Top $20 \mathrm{important}$ features are shown in figures. Feature importance of a RF-method model, $\mathbf{b}$ XGBoost model, and $\mathbf{c}$ ANN model in patients with RA. Feature importance of $\mathbf{d}$ RF-method model, e XGBoost model, and $\mathbf{f}$ ANN model in patients with AS. WBC, white cell count; BMI, body mass index; Plt, platelet; $\mathrm{Hb}$, hemoglobin; $\mathrm{Hct}$, hematocrit; DM, diabetes mellitus; anti-CCP, anti-cyclic citrullinated protein; ILD, interstitial lung disease; MTX, methotrexate; TACRO, tacrolimus; LEFL, leflunomide; SSZ, sulfasalazine 

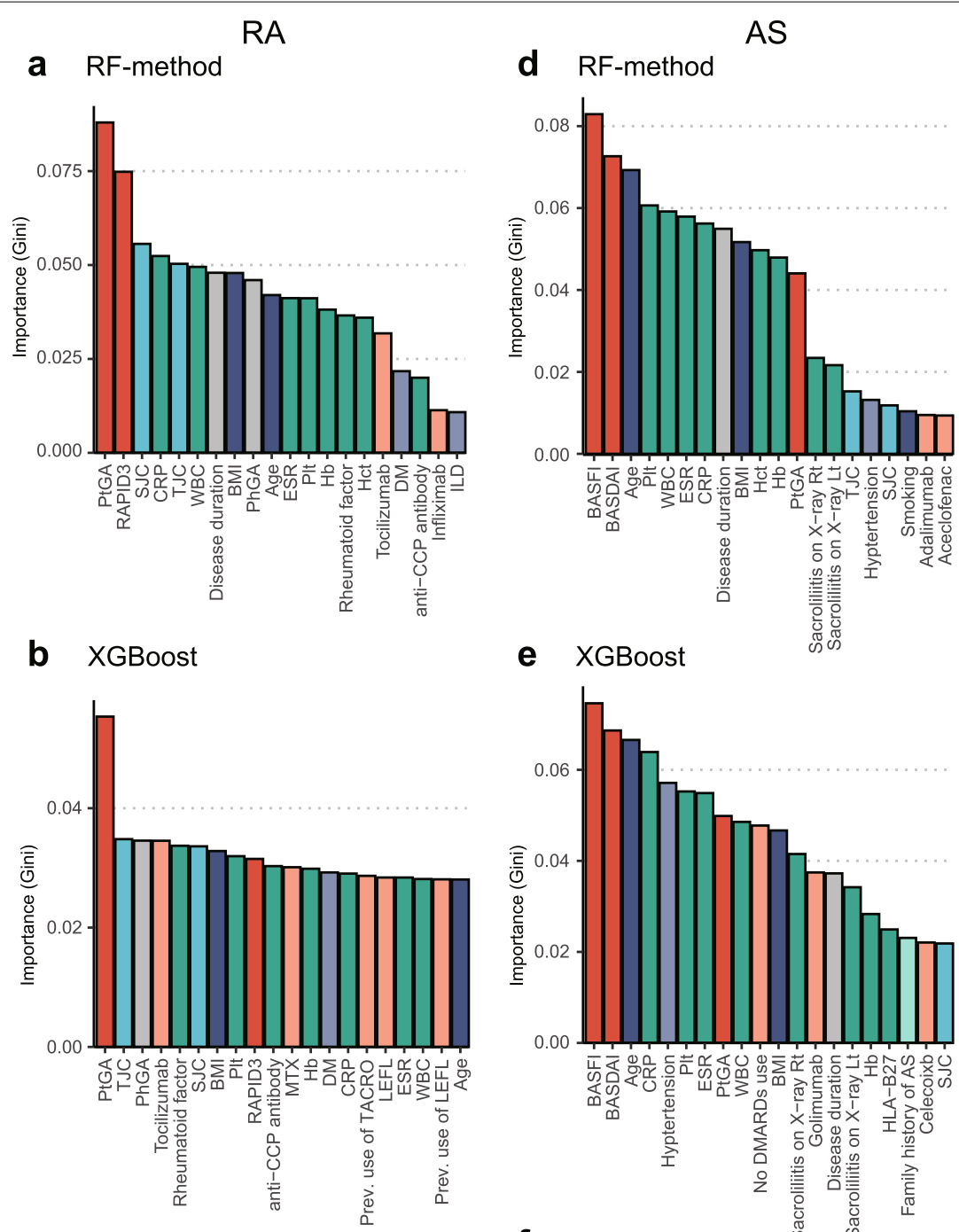

e XGBoost

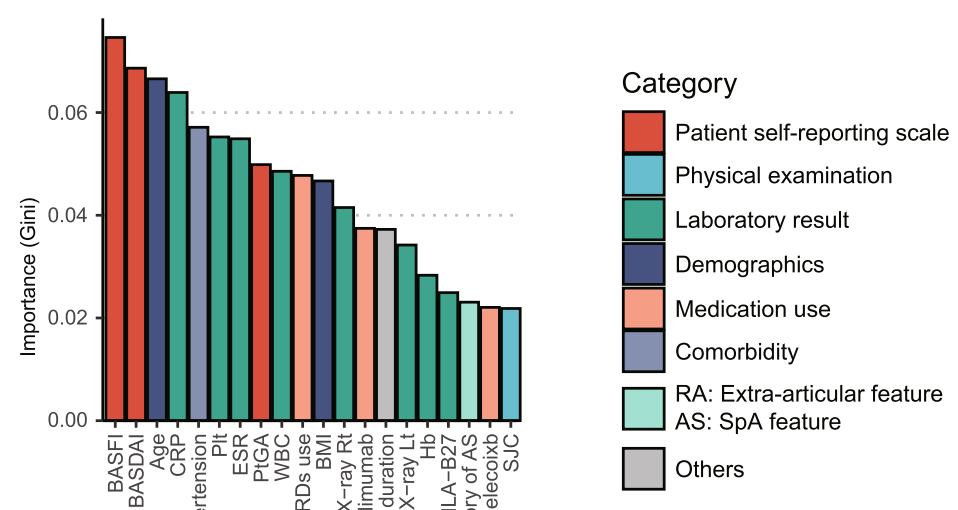

C ANN

f ANN
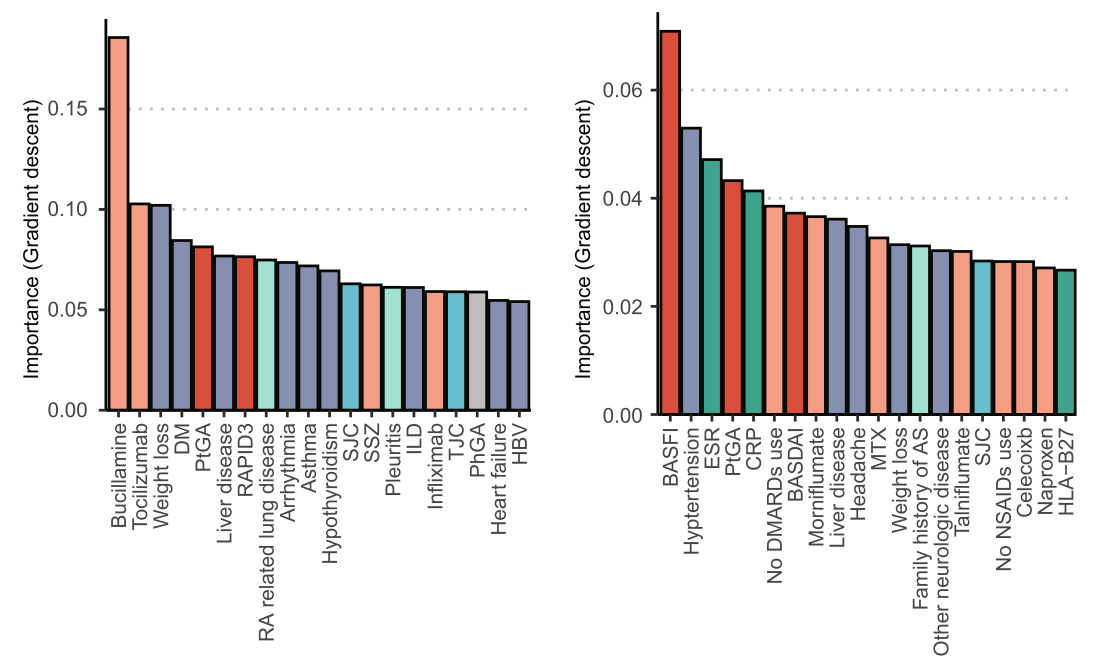

Fig. 4 (See legend on previous page.) 
accurately than the conventional statistical method, which is logistic regression. However, machine learning models to predict treatment responses of biologic agents in AS patients are not superior in contrast to RA. According to the feature importance analysis, patient self-reporting scales were the most important input features in both diseases. Only a few previous studies have been published to predict treatment responses to biologic agents in RA patients [16,34]. However, the present study includes a more detailed feature importance analysis than previous studies. Furthermore, this is the first attempt to predict the treatment responses of bDMARDs in AS patients.

We implemented various machine learning methods to predict treatment responses, including RF-method, XGBoost, ANN, and SVM. Both RF-method and XGBoost are ensemble models that consist of numerous small decision trees. RF-method is based on a bagging algorithm, and XGBoost is based on a gradient boosting algorithm. Although SVM is relatively older, it exhibits a satisfactory performance in simple image classification with little computational burden. ANNs are gradually gaining popularity as they obtain successful results in various fields, such as image classification. However, decision-tree-based algorithms show better performance in certain circumstances, such as small, tabular data [35]. RF-method showed better prediction performance than ANN in RA patients in this study. In addition, the optimal ANN prediction model had only one (RA) or two (AS) hidden layers, which are too shallow to obtain the advantage of ANN. Therefore, our input data seemed unsuitable for the ANN. This could be because of the relatively small size of the input data.

RF-method showed better prediction performance than logistic regression in patients with RA but not in those with AS. In addition, the prediction performance of the various models was lower in AS patients. Determining the exact reason requires further research and is beyond the scope of this study, although some speculation can be made. The number of data points was slightly smaller in AS; however, the difference was only $5-10 \%$ of all patients. The number of input features of the AS was higher than that of the RA. RA had a more unbalanced responder/non-responder proportion, which generally had a negative effect on machine learning results. Thus, the differences in the prediction performance were unlikely because of the structure of the input dataset. If so, we could assume that the input features were insufficient to predict the treatment response of bDMARDs in patients with AS. Heritability analysis implied that AS has more genetic factors than RA, with higher heritability of approximately $80-90 \%$ [36-39] vs. $50-60 \%$ $[40,41]$ in AS and RA, respectively. Previous studies have shown that genetic features could affect the response of bDMARDs in patients with AS [42, 43]. In addition, there have been pilot studies of transcriptome analysis [44, 45] to predict the responses of bDMARDs in patients with AS. Therefore, multi-omics data, including genetics and transcriptomics, may improve prediction performance.

Feature importance analysis can provide insights into clinical factors. In this study, machine learning models revealed that the patient self-reporting scales, PtGA and BASFI in RA and AS patients, respectively, were the most important factors for predicting treatment responses. It is quite surprising because they are more important than more objective clinical features, such as laboratory results (ESR and CRP) and physical examination (SJC and TJC). Previous studies reported patient self-reporting scales, such as RAPID3 [46] or BASFI [47] as predictors of bDMARD treatment. However, their relative importance compared with other objective disease activities or functional measures has not been studied. In addition, given that the results of feature importance were similar except for ANN in RA patients, which had inferior performances, the result of the feature important analysis was robust.

The prediction models were trained for each medication use separately. However, the performance of prediction models using RF-method was not superior to that of logistic regression models in each medication dataset. Only the prediction model of adalimumab users in patients with AS using RF-method had a borderline superior result to the logistic regression model. The results of the feature importance analysis for each medication user were not consistent. Again, only the model of adalimumab patients in patients with AS showed similar results to the entire cohort in the feature importance analysis. Adalimumab users in patients with AS occupied the largest patient group with 253 individuals, while the other cohorts comprised less than 200 patients. Therefore, the size of the patient group must be an important factor in generating a proper predictive model, and approximately 250 people could be the lower limit of size.

However, our approach had some limitations. First, even though we divided part of the dataset by the region of hospitals as an independent test dataset and did not participate in any part of the training machine learning model, the validation cohort was not retrieved from a completely different cohort. However, forty-five hospitals were involved in the KOBIO cohorts, and each hospital had an independent enrollment process, assessment physician, and laboratory institution. Thus, we expect that pre-divided test dataset represents an independent cohort. Second, all participants were Koreans, therefore we do not assure that the models we generated showed similar results in other populations. When applied to 
other populations, new patient data or feature selection may be required in advance.

\section{Conclusions}

In conclusion, we developed several machine learning models that could predict the treatment responses of biologic agents in patients with RA and AS. The best-performing model was trained using RF-method in patients with RA. The model performs better than the conventional statistical method, logistic regression. Given the input clinical features, machine learning models have no advantages compared to a logistic regression model in patients with AS. Feature importance analysis shows that patient self-reporting scales, PtGA and BASFI in RA and AS patients, respectively, are the most important input features for machine learning prediction models.

\begin{abstract}
Abbreviations
ACR: American College of Rheumatology; ACR20: ACR 20\% improvement response criteria; ANN: Artificial neural network; AS: Ankylosing spondylitis; ASAS: Assessment of Spondyloarthritis International Society; ASAS20: ASAS 20\% improvement response criteria; AUC: Area under curve; BASDAI: Bath Ankylosing Spondylitis Disease Activity Index; BASFI: Bath Ankylosing Spondylitis Functional Index; bDMARDs: Biologic disease-modifying antirheumatic drugs; Cl: Confidence interval; CRP: C-reactive protein; CSDMARDs: Vonventional synthetic disease-modifying rheumatic drugs; ESR: Erythrocyte sedimentation rate; EULAR: European League Against Rheumatism; KOBIO: Korean College of Rheumatology Biologics and Targeted Therapy; PhGA: Physician global assessment of disease activity; PtGA: Patient global assessment of disease activity; RA: Rheumatoid arthritis; RAPID3: Routine assessment of patient index data 3; ROC: Receiver operating characteristic curve; SJC: Swollen joint count; SVM: Support vector machine; TJC: Tender joint count; XGBoost: Extreme gradient boosting.
\end{abstract}

\section{Supplementary Information}

The online version contains supplementary material available at https://doi. org/10.1186/s13075-021-02635-3.

Additional file 1: Text S1. Detailed methods for dividing test dataset and feature importance analysis, and additional analysis with features reported from the feature importance analysis.

Additional file 2: Figure S1-3. Prediction performance of machine learning methods by varying hyperparameters in RA patients. Figure S4-6. Prediction performance of machine learning methods by varying hyperparameters in AS patients. Figure S7. Performance of RF-method and logistic regression models in RA patients divided by the type of bDMARDs. Figure $\mathbf{S 8}$. Result of the feature importance analysis of the best-performing RF-method model in RA patients divided by the type of bDMARDs. Figure S9. Performance of RF-method and logistic regression models in AS patients divided by the type of bDMARDs. Figure S10. Feature importance analysis of the best-performing RF-method model in AS patients divided by the type of bDMARDs. Figure S11. Correlation analysis between input features that reported the feature importance analysis. Figure S12. Linear regression analysis with dot plot results for input features. Figure S13. Prediction performances of logistic regression models using patient self-reported scales and their combinations.

Additional file 3: Table S1. List of input features. Table S2. Chosen hyperparameter sets for each model.

\section{Acknowledgements}

The authors would like to thank Mingyun Bae, PhD candidate of the omics lab, KAIST, for his help in analyzing feature importance.

\section{Authors' contributions}

Conceptualization: Seulkee Lee, Seonyoung Kang, Yeonghee Eun, Hong-Hee Won, Hyungjin Kim, Jaejoon Lee, Eun-Mi Koh, Hoon-Suk Cha. Data curation: Seulkee Lee. Formal analysis: Seulkee Lee. Investigation: Seulkee Lee. Methodology: Seulkee Lee, Hong-Hee Won, Hoon-Suk Cha. Project administration: Seulkee Lee, Hoon-Suk Cha. Supervision: Hong-Hee Won, Hoon-Suk Cha. Visualization: Seulkee Lee. Writing - original draft: Seulkee Lee. Writing - review and editing: Seulkee Lee, Seonyoung Kang, Yeonghee Eun, Hong-Hee Won, Hyungjin Kim, Jaejoon Lee, Eun-Mi Koh, Hoon-Suk Cha. The author(s) read and approved the final manuscript.

\section{Funding}

None.

\section{Availability of data and materials}

All data generated or analyzed during this study are included in this published article. Our training codes and generated prediction models have been made publicly accessible (https://github.com/SeulkeeLee123/KOBIO_biologics).

\section{Declarations}

\section{Ethics approval and consent to participate}

This research complied with the principles of the Declaration of Helsinki. All patients provided written consent to participate in the registry, and the data collection form and study protocol were approved by the institutional review boards or local ethics committees at each participating center. The IRB of Samsung Medical Center approved the study (SMC 2021-04-083).

\section{Competing interests}

None.

\section{Author details}

${ }^{1}$ Department of Medicine, Samsung Medical Center, Sungkyunkwan University School of Medicine, 81 Irwon-ro, Gangnam-gu, Seoul 06351, Republic of Korea. ${ }^{2}$ Samsung Advanced Institute for Health Sciences \& Technology (SAIHST), Sungkyunkwan University, Samsung Medical Center, Seoul, Republic of Korea.

Received: 14 May 2021 Accepted: 27 September 2021

Published online: 09 October 2021

\section{References}

1. Dixon WG, Symmons DP, Lunt M, Watson KD, Hyrich KL. British Society for Rheumatology Biologics Register Control Centre C, et al. Serious infection following anti-tumor necrosis factor alpha therapy in patients with rheumatoid arthritis: lessons from interpreting data from observational studies. Arthritis Rheum. 2007;56(9):2896-904.

2. Hetland ML, Christensen IJ, Tarp U, Dreyer L, Hansen A, Hansen IT, et al. Direct comparison of treatment responses, remission rates, and drug adherence in patients with rheumatoid arthritis treated with adalimumab, etanercept, or infliximab: results from eight years of surveillance of clinical practice in the nationwide Danish DANBIO registry. Arthritis Rheum. 2010;62(1):22-32.

3. Coates LC, Cawkwell LS, Ng NW, Bennett AN, Bryer DJ, Fraser AD, et al. Real life experience confirms sustained response to long-term biologics and switching in ankylosing spondylitis. Rheumatology (Oxford). 2008;47(6):897-900.

4. Lee JW, Kang JH, Yim YR, Kim JE, Wen L, Lee KE, et al. Predictors of Switching Anti-Tumor Necrosis Factor Therapy in Patients with Ankylosing Spondylitis. PLoS One. 2015;10(7):e0131864.

5. Moreno M, Gratacos J, Torrente-Segarra V, Sanmarti R, Morla R, Pontes C, et al. Withdrawal of infliximab therapy in ankylosing spondylitis in persistent clinical remission, results from the REMINEA study. Arthritis Res Ther. 2019;21(1):88. 
6. Aletaha D, Kapral T, Smolen JS. Toxicity profiles of traditional disease modifying antirheumatic drugs for rheumatoid arthritis. Ann Rheum Dis. 2003;62(5):482-6.

7. Antoni C, Braun J. Side effects of anti-TNF therapy: current knowledge. Clin Exp Rheumatol. 2002;20(6 Suppl 28):S152-7.

8. de la Torre I, Valor L, Nieto JC, Hernandez-Florez D, Martinez L, Gonzalez CM, et al. Anti-TNF treatments in rheumatoid arthritis: economic impact of dosage modification. Expert Rev Pharmacoecon Outcomes Res. 2013:13(3):407-14.

9. Kim Y, Kim G-T, Suh YS, Kim H-O, Lee H-N, Lee S-G. The Impact of the Amendment of the Korean National Health Insurance Reimbursement Criteria for Anti-tumor Necrosis Factor-a Agents on Treatment Pattern, Clinical Response and Persistence in Patients With Rheumatoid Arthritis. J Rheum Dis. 2020;27(3):159-67.

10. Glintborg B, Ostergaard M, Krogh NS, Tarp U, Manilo N, Loft AG, et al. Clinical response, drug survival and predictors thereof in 432 ankylosing spondylitis patients after switching tumour necrosis factor alpha inhibitor therapy: results from the Danish nationwide DANBIO registry. Ann Rheum Dis. 2013;72(7):1149-55.

11. Katchamart W, Johnson S, Lin HJ, Phumethum V, Salliot C, Bombardier C. Predictors for remission in rheumatoid arthritis patients: A systematic review. Arthritis Care Res. 2010;62(8):1128-43.

12. Kakadiaris IA, Vrigkas M, Yen AA, Kuznetsova T, Budoff M, Naghavi M. Machine Learning Outperforms ACC / AHA CVD Risk Calculator in MESA. J Am Heart Assoc. 2018;7(22):e009476.

13. Rutledge RB, Chekroud AM, Huys QJ. Machine learning and big data in psychiatry: toward clinical applications. Curr Opin Neurobiol. 2019;55:152-9.

14. Shung DL, Au B, Taylor RA, Tay JK, Laursen SB, Stanley AJ, et al. Validation of a Machine Learning Model That Outperforms Clinical Risk Scoring Systems for Upper Gastrointestinal Bleeding. Gastroenterology. 2020;158(1):160-7.

15. Wu J, Roy J, Stewart WF. Prediction modeling using EHR data: challenges, strategies, and a comparison of machine learning approaches. Med Care. 2010:48(6 Suppl):S106-13.

16. Guan Y, Zhang H, Quang D, Wang Z, Parker SCJ, Pappas DA, et al. Machine Learning to Predict Anti-Tumor Necrosis Factor Drug Responses of Rheumatoid Arthritis Patients by Integrating Clinical and Genetic Markers. Arthritis Rheum. 2019;71(12):1987-96.

17. Lee S, Eun Y, Kim H, Cha HS, Koh EM, Lee J. Machine learning to predict early TNF inhibitor users in patients with ankylosing spondylitis. Sci Rep. 2020;10(1):20299.

18. Choi IA. Comparison of the Disease Activity Score-28 Based on the Erythrocyte Sedimentation Rate and C-reactive Protein in Rheumatoid Arthritis. J Rheuma Dis. 2017;24(5):287-92.

19. Felson DT, Anderson JJ, Boers M, Bombardier C, Furst D, Goldsmith C, et al. American College of Rheumatology. Preliminary definition of improvement in rheumatoid arthritis. Arthritis Rheum. 1995;38(6):727-35.

20. Anderson JJ, Baron G, van der Heijde D, Felson DT, Dougados M. Ankylosing spondylitis assessment group preliminary definition of short-term improvement in ankylosing spondylitis. Arthritis Rheum. 2001;44(8):1876-86.

21. Baeten D, Sieper J, Braun J, Baraliakos X, Dougados M, Emery P, et al. Secukinumab, an Interleukin-17A Inhibitor, in Ankylosing Spondylitis. N Engl J Med. 2015;373(26):2534-48.

22. Burmester GR, Kremer JM, Van den Bosch F, Kivitz A, Bessette L, Li Y, et al. Safety and efficacy of upadacitinib in patients with rheumatoid arthritis and inadequate response to conventional synthetic disease-modifying anti-rheumatic drugs (SELECT-NEXT): a randomised, double-blind, placebo-controlled phase 3 trial. Lancet. 2018;391(10139):2503-12.

23. Taylor PC, Keystone EC, van der Heijde D, Weinblatt ME, Del Carmen ML, Reyes Gonzaga J, et al. Baricitinib versus Placebo or Adalimumab in Rheumatoid Arthritis. N Engl J Med. 2017;376(7):652-62.

24. Breiman L. Random forests. Mach Learn. 2001:45(1):5-32.

25. Chen T, Guestrin C. Xgboost: A scalable tree boosting system. 2016. https://arxiv.org/abs/1603.02754.

26. Jain AK, Mao JC, Mohiuddin KM. Artificial neural networks: a tutorial. Computer. 1996;29(3):31.

27. Kim JH. Estimating classification error rate: Repeated cross-validation, repeated hold-out and bootstrap. Comput Stat Data Anal. 2009;53(11):3735-45.
28. Raschka S. Model evaluation, model selection, and algorithm selection in machine learning. 2018. https://arxiv.org/abs/1811.12808.

29. Shrikumar A, Greenside P, Kundaje A. Learning Important Features Through Propagating Activation Differences. 2017. https://arxiv.org/abs/ 1704.02685. Accessed 1 April 2021.

30. Yousefi S, Amrollahi F, Amgad M, Dong C, Lewis JE, Song C, et al. Predicting clinical outcomes from large scale cancer genomic profiles with deep survival models. Sci Rep. 2017;7(1):11707.

31. R Core Team. R: A language and environment for statistical computing. 2013

32. Pedregosa F, Varoquaux G, Gramfort A, Michel V, Thirion B, Grisel O, et al. Scikit-learn: Machine Learning in Python. J Mach Learn Res. 2011;12:2825-30.

33. Abadi M, Barham P, Chen J, Chen Z, Davis A, Dean J, et al. Tensorflow: A system for large-scale machine learning. 2016. https://arxiv.org/abs/1605. 08695. Accessed 1 April 2021.

34. Tao W, Concepcion AN, Vianen M, Marijnissen ACA, Lafeber F, Radstake T, et al. Multiomics and Machine Learning Accurately Predict Clinical Response to Adalimumab and Etanercept Therapy in Patients With Rheumatoid Arthritis. Arthritis Rheum. 2021;73(2):212-22.

35. Han T, Jiang D, Zhao Q, Wang L, Yin K. Comparison of random forest, artificial neural networks and support vector machine for intelligent diagnosis of rotating machinery. Trans Inst Meas Control. 2017;40(8):2681-93.

36. Brown MA. Breakthroughs in genetic studies of ankylosing spondylitis. Rheumatology (Oxford). 2008;47(2):132-7.

37. Brown MA, Kennedy LG, MacGregor AJ, Darke C, Duncan E, Shatford JL, et al. Susceptibility to ankylosing spondylitis in twins: the role of genes, HLA, and the environment. Arthritis Rheum. 1997;40(10):1823-8.

38. Morin M, Hellgren K, Frisell T. Familial aggregation and heritability of ankylosing spondylitis - a Swedish nested case-control study. Rheumatology (Oxford). 2020:59(7):1695-702.

39. Pedersen O, Svendsen A, Ejstrup L, Skytthe A, Harris J, Junker P. Heritability estimates on ankylosing spondylitis. Clin Exp Rheumatol. 2006;24(4):463.

40. Frisell T, Holmqvist M, Kallberg H, Klareskog L, Alfredsson L, Askling J. Familial risks and heritability of rheumatoid arthritis: role of rheumatoid factor/anti-citrullinated protein antibody status, number and type of affected relatives, sex, and age. Arthritis Rheum. 2013;65(11):2773-82.

41. Karami J, Aslani S, Jamshidi A, Garshasbi M, Mahmoudi M. Genetic implications in the pathogenesis of rheumatoid arthritis; an updated review. Gene. 2019;702:8-16.

42. Seitz M, Wirthmuller U, Moller B, Villiger PM. The -308 tumour necrosis factor-alpha gene polymorphism predicts therapeutic response to TNFalpha-blockers in rheumatoid arthritis and spondyloarthritis patients. Rheumatology (Oxford). 2007;46(1):93-6.

43. Tong Q, Zhao DB, Bajracharya P, Xu X, Kong RN, Zhang J, et al. TNF-alpha -857 and -1031 polymorphisms predict good therapeutic response to TNF-alpha blockers in Chinese Han patients with ankylosing spondylitis. Pharmacogenomics. 2012;13(13):1459-67.

44. Harrison SR, Burska AN, Emery P, Marzo-Ortega H, Ponchel F. Interferon-related gene expression in response to TNF inhibitor treatment in ankylosing spondylitis patients: a pilot study. Rheumatology (Oxford). 2021;60(8):3607-16.

45. Wang XB, Ellis JJ, Pennisi DJ, Song X, Batra J, Hollis K, et al. Transcriptome analysis of ankylosing spondylitis patients before and after TNFalpha inhibitor therapy reveals the pathways affected. Genes Immun. 2017;18(3):184-90.

46. Curtis JR, Churchill M, Kivitz A, Samad A, Gauer L, Gervitz L, et al. A Randomized Trial Comparing Disease Activity Measures for the Assessment and Prediction of Response in Rheumatoid Arthritis Patients Initiating Certolizumab Pegol. Arthritis Rheum. 2015;67(12):3104-12.

47. Vastesaeger N, van der Heijde D, Inman RD, Wang Y, Deodhar A, Hsu B, et al. Predicting the outcome of ankylosing spondylitis therapy. Ann Rheum Dis. 2011;70(6):973-81.

\section{Publisher's Note}

Springer Nature remains neutral with regard to jurisdictional claims in published maps and institutional affiliations. 